\section{Controlling nanostructures}

SIR - Röder et al. ${ }^{1}$ report nanometrescale structures built by deposition of diffusing particles that aggregate on surfaces. We have developed a microscopic model that mimics the same process, and produces morphologies that remarkably resemble the experimental structures.

The model is defined as follows: (1) Deposition. Particles are deposited at randomly-chosen positions of the surface at a flux $F$ per lattice site per unit time. (2) Diffusion. A cluster of connected particles is chosen at random and moved north east, south or west by one lattice constant per unit time with a probability proportional to its mobility, which is given by $D_{s}=D_{1} s^{-\gamma}$, where $s$ is the number of particles in the cluster, $D_{1}$ is the diffusion constant of the monomers and $\gamma$ characteon its size. (3) Aggregation. If two particles come to occupy neighbouring sites, they stick irreversibly. comparison with the experimental data in ref. 1. There are no free parameters provided we introduce the experimental values for the flux and the diffusion constant. The diffusion constant of the monomers is given by $D_{1}(T)=D_{0} \exp (-$ $\left.E_{d} / k T\right)$ with $E_{d}=0.14 \mathrm{eV}$ (ref. 1) and $D_{0}$ $=5 \times 10^{11}$ (ref. 2). Using the experimental values of the fluxes, we find $F / D_{1}=10^{3}$ corresponds to Fig. $1 a$ of ref. 1 , and $F / D_{1}=10^{-10}$ to Fig. $1 d$. The figures here show results of the model with these flux values; note that the morphologies compare well with Fig. $1 a$ and $d$ of ref. 1 .

In general, our model allows one to distinguish the effects of deposition, diffusion and aggregation. We find that tuning

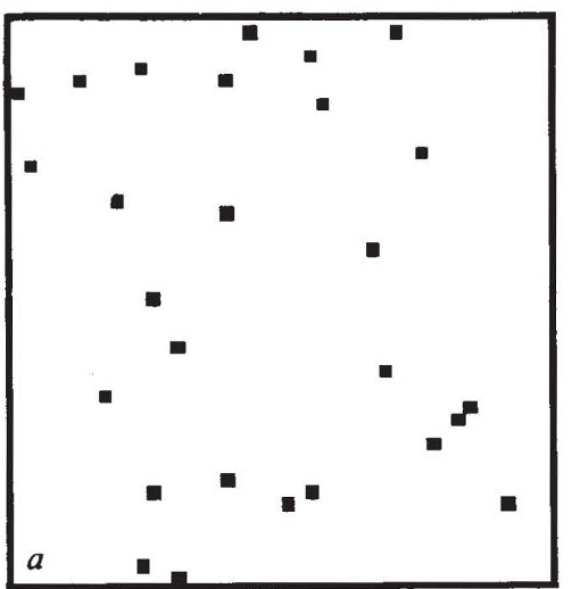
rizes how the mobility of a cluster depends

The model can be tested by explicit
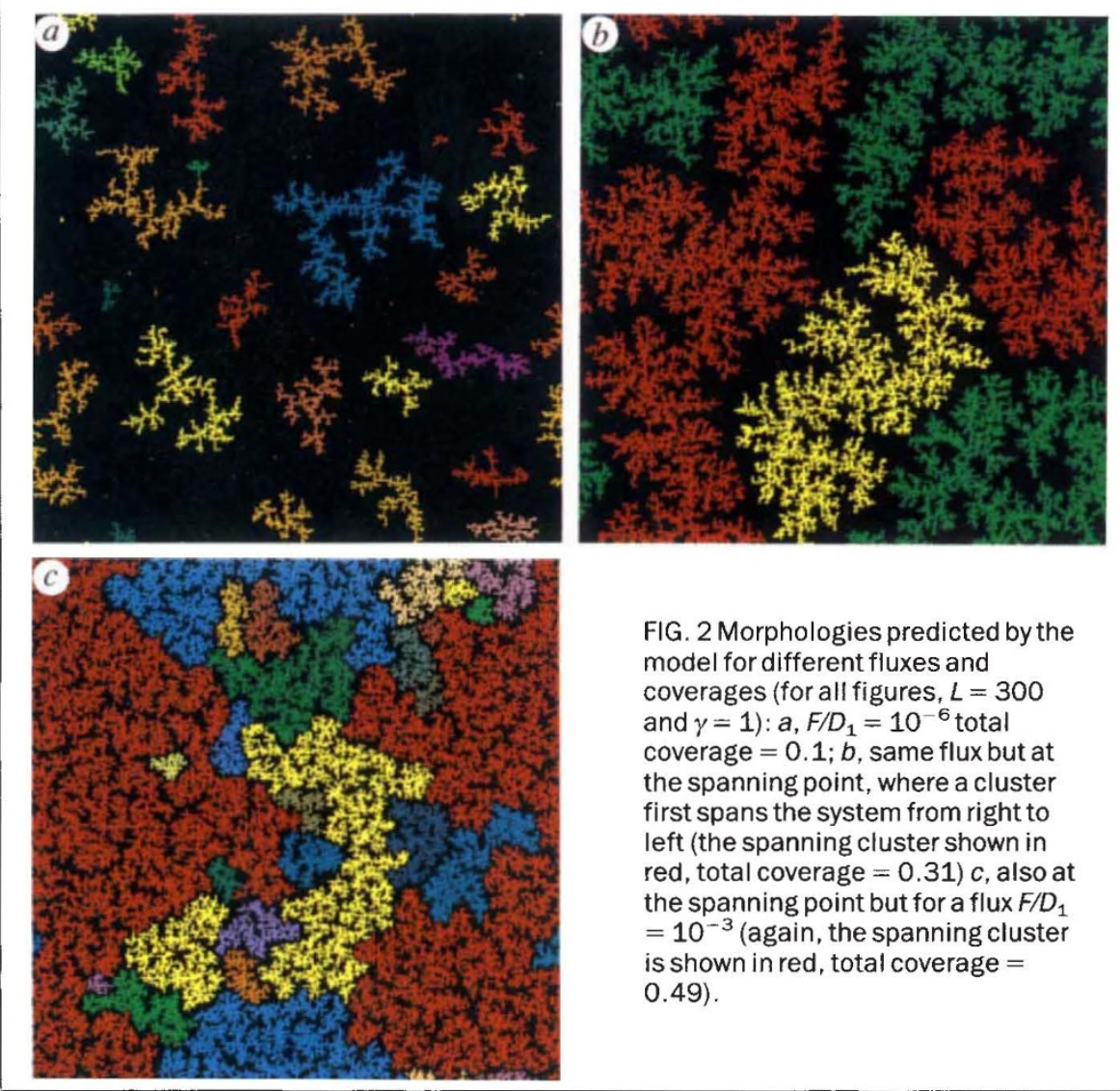

FIG. 2 Morphologies predicted by the model for different fluxes and coverages (for all figures, $L=300$ and $\gamma=1): a, F / D_{1}=10^{-6}$ total coverage $=0.1 ; b$, same flux but at the spanning point, where a cluster first spans the system from right to left (the spanning cluster shown in red, total coverage $=0.31$ ) $c$, also at the spanning point but for a flux $F / D_{1}$ $=10^{-3}$ (again, the spanning cluster is shown in red, total coverage $=$ 0.49).

the relative strength of, for example, deposition and diffusion, generates a rich range of morphologies - including diffusion limited aggregation (DLA), clustercluster aggregation, and percolation ${ }^{3}$. The length- and timescales characterizing these morphologies depend on experimentally controlled parameters like deposition flux and diffusion constant, raising the possibility that the model could be used for a controlled design of nonostructure morphologies. Indeed, the model

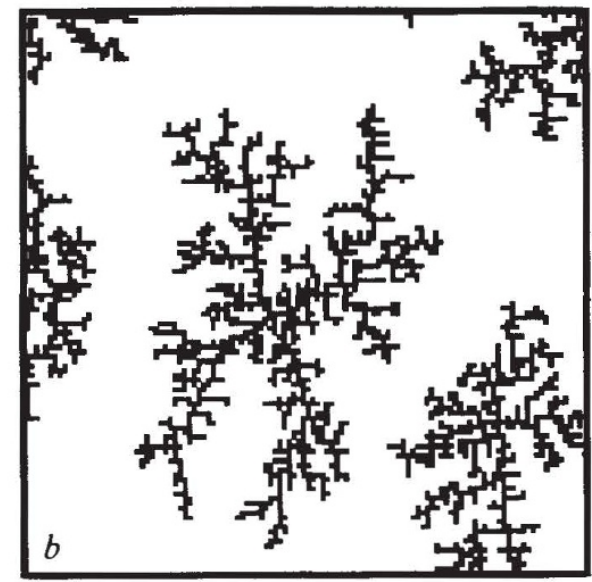

FIG. 1 Morphologies obtained in the present model for two different values of $F, D_{1}$ and total surface coverage all chosen to correspond to the experimental parameters used in obtaining the data shown in Fig. $1 a$ and $d$ of ref. 1. $a, F / D_{1}=10^{3}$, and total coverage of $0.012 . b, F / D_{1}=10^{-10}$ and total coverage of 0.12 . The simulation lattice had $200 \times 200$ sites; the portion shown here corresponds to Fig. $1 \mathrm{a}$ and $d$ of ref. 1, which are also a portion of the total experimental system. We set $\gamma=10$ (large clusters rarely move $-J$. P. Bucher, personal communication). makes specific predictions, for example that the typical size of the DLA-like structures scales as $\left(F / D_{1}\right)^{-1 / 4}$.

Varying the model parameters produces a rich range of morphologies (Fig. 2), and the model may be useful in a variety of situations where diffusion and aggregation occur in the presence of continuous deposition. The model was originally motivated by thin-film deposition experiments in which not isolated atoms but rather aggregates (compact, spherical molecules of about 5-nm diameter, containing about 2,000 atoms) are deposited on a surface ${ }^{4}$. The morphologies of Figs 1 and 2 also resemble experimental images obtained by such low-energy cluster beam deposition experiments on substrates maintained at low temperatures (compare Fig. $2 a$ to Fig. 3 of ref. 4).

Pablo Jensen, Albert-László Barabási, Hernán Larralde, Shlomo Havlin

\section{\& H. Eugene Stanley}

Center for Polymer Studies and Physics

Department, Boston University, Boston, Massachusetts 02215, USA

Permanent addresses: Département de Physique des Matér iaux, Université Claude Bernard Lyon-1, Villeurbanne Cedex. France (P.J.); Physics Department. Bar Itan University, Ramat Gan, Israel (S.H.)

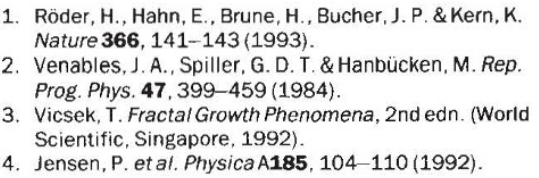

